\title{
Night Time
}

National Cancer Institute

\section{Source}

National Cancer Institute. Night Time. NCI Thesaurus. Code C65001.

The time in every 24 hour period when it is dark. 